\title{
TWIN ROMAN DOMINATION NUMBER OF A DIGRAPH
}

\author{
H. ABDOLLAHZADEH AHANGAR, J. AMJADI, S.M. SHEIKHOLESLAMI, \\ V. SAMODIVKIN, AND L. VOLKMANN
}

Received 25 March, 2014

\begin{abstract}
Let $D$ be a finite and simple digraph with vertex set $V(D)$. A twin Roman dominating function (TRDF) on $D$ is a labeling $f: V(D) \rightarrow\{0,1,2\}$ such that every vertex with label 0 has an in-neighbor and out-neighbor with label 2. The weight of a TRDF $f$ is the value $\omega(f)=$ $\sum_{v \in V(D)} f(v)$. The twin Roman domination number of a digraph $D$, denoted by $\gamma_{R}^{*}(D)$, equals the minimum weight of a TRDF on $D$. In this paper we initiate the study of the twin Roman domination number in digraphs. In particular, we present sharp bounds for $\gamma_{R}^{*}(D)$ and determine the exact value of the twin Roman domination number for some classes of digraphs.
\end{abstract}

2010 Mathematics Subject Classification: 05 C69

Keywords: twin Roman dominating function, twin Roman domination number, Roman dominating function, Roman domination number, digraph

\section{INTRODUCTION}

Let $D$ be a finite simple digraph with vertex set $V(D)=V$ and arc set $A(D)=$ $A$. A digraph without directed cycles of length 2 is an oriented graph. The order $n=n(D)$ of a digraph $D$ is the number of its vertices. We write $d_{D}^{+}(v)$ for the out-degree of a vertex $v$ and $d_{D}^{-}(v)$ for its in-degree. The minimum and maximum in-degree and minimum and maximum out-degree of $D$ are denoted by $\delta^{-}=\delta^{-}(D)$, $\Delta^{-}=\Delta^{-}(D), \delta^{+}=\delta^{+}(D)$ and $\Delta^{+}=\Delta^{+}(D)$, respectively. The minimum degree $\delta(D)$ of a digraph $D$ is defined as the minimum of all in-degrees and all out-degrees of vertices in $D$ and the maximum degree $\Delta(D)$ of a digraph $D$ is defined as the maximum of all in-degrees and all out-degrees of vertices in $D$. If $u v$ is an arc of $D$, then we also write $u \rightarrow v$, and we say that $v$ is an out-neighbor of $u$ and $u$ is an in-neighbor of $v$. For a vertex $v$ of a digraph $D$, we denote the set of in-neighbors and out-neighbors of $v$ by $N^{-}(v)=N_{D}^{-}(v)$ and $N^{+}(v)=N_{D}^{+}(v)$, respectively. If $X \subseteq$ $V(D)$, then $D[X]$ is the subdigraph induced by $X$. If $X \subseteq V(D)$ and $v \in V(D)$, then $E(X, v)$ is the set of arcs from $X$ to $v$. Consult [8] for the notation and terminology which are not defined here. For a real-valued function $f: V(D) \longrightarrow \mathbb{R}$ the weight of $f$ is $w(f)=\sum_{v \in V(D)} f(v)$, and for $S \subseteq V(D)$, we define $f(S)=\sum_{v \in S} f(v)$, so $w(f)=f(V(D))$. 
A vertex $u$ in a digraph $D$ out-dominates itself and all vertices $v$ such that $u v$ is an arc of $D$, similarly, $u$ in-dominates both itself and all vertices $w$ such that $w u$ is an $\operatorname{arc}$ of $D$. A set $S$ of vertices of $D$ is a twin dominating set of $D$ if every vertex of $D$ is out-dominated by a vertex of $S$ and in-dominated by a vertex of $S$. The twin domination number $\gamma^{*}(D)$ is the cardinality of a minimum twin dominating set. A $\gamma^{*}(D)$-function is a twin dominating function of $D$ with weight $\gamma^{*}(D)$. The twin domination, was introduced by Chartrand, Dankelmann, Schultz, and Swart [3] and has been studied by several authors (see $[1,2,6]$ ).

A Roman dominating function (RDF) on a digraph $D$ is a function $f: V \longrightarrow$ $\{0,1,2\}$ satisfying the condition that every vertex $v$ for which $f(v)=0$ has a inneighbor $u$ for which $f(u)=2$. The weight of an RDF $f$ is the value $\omega(f)=$ $\sum_{v \in V} f(v)$. The Roman domination number of a digraph $D$, denoted by $\gamma_{R}(D)$, equals the minimum weight of an RDF on D. A $\gamma_{R}(D)$-function is a Roman dominating function of $D$ with weight $\gamma_{R}(D)$. The Roman domination for digraphs was introduced by Kamaraj and Hemalatha [5] and investigated in [7].

A twin Roman dominating function (TRDF) on $D$ is a Roman dominating function of $D$ such that every vertex with label 0 has an out-neighbor with label 2 . The twin Roman domination number of a digraph $D$, denoted by $\gamma_{R}^{*}(D)$, equals the minimum weight of a TRDF on $D$. A $\gamma_{R}^{*}(D)$-function is a twin Roman dominating function of $D$ with weight $\gamma_{R}^{*}(D)$. A twin Roman dominating function $f: V \longrightarrow\{0,1,2\}$ can be represented by the ordered partition $\left(V_{0}, V_{1}, V_{2}\right)$ (or $\left(V_{0}^{f}, V_{1}^{f}, V_{2}^{f}\right)$ to refer $f$ ) of $V$, where $V_{i}=\{v \in V \mid f(v)=i\}$. In this representation, its weight is $\omega(f)=$ $\left|V_{1}\right|+2\left|V_{2}\right|$. Since $V_{1}^{f} \cup V_{2}^{f}$ is a twin dominating set when $f$ is a TRDF, and since placing weight 2 at the vertices of a twin dominating set yields a TRDF, we have

$$
\gamma^{*}(D) \leq \gamma_{R}^{*}(D) \leq 2 \gamma^{*}(D) \text {. }
$$

Obviously the function $f=(\varnothing, V(D), \varnothing)$ is a TRDF of $D$ which implies that

$$
\gamma_{R}^{*}(D) \leq n
$$

Our purpose in this paper is to establish some sharp bounds for the twin Roman domination number of a digraph.

We make use of the following results in this paper.

Theorem 1 ([3]). Let $D$ be a digraph of order $n$ and minimum degree $\delta(D) \geq 1$. Then,

$$
\gamma^{*}(D) \leq\left\lfloor\frac{2 n}{3}\right\rfloor
$$

The proof of the following observations are straightforward and therefore omitted.

Observation 1. Let $D$ be a digraph on $n$ vertices. Then

(i) If $\gamma_{R}^{*}(D)=n$ then for any $\gamma_{R}^{*}$-function $f=\left(V_{0}, V_{1}, V_{2}\right)$ on $D,\left|V_{0}\right|=\left|V_{2}\right|$.

(ii) If $\left|V_{0}\right|=\left|V_{2}\right|$ for some $\gamma_{R}^{*}$-function $f=\left(V_{0}, V_{1}, V_{2}\right)$ on $D$, then $\gamma_{R}^{*}(D)=n$. 
Observation 2. Let $D$ be a digraph and $f=\left(V_{0}^{f}, V_{1}^{f}, V_{2}^{f}\right)$ a TRDF on D.

(i) If $x, y, z \in V_{1}, x \rightarrow y, y \rightarrow x, y \rightarrow z$ and $z \rightarrow y$ then $g=\left(V_{0}^{f} \cup\{x, z\} ; V_{1}^{f}-\right.$ $\left.\{x, y, z\} ; V_{2}^{f} \cup\{y\}\right)$ is a TRDF on D with $w(g)=w(f)-1$.

(ii) If $x \in V_{2}, y \in V_{1}, x \rightarrow y$ and $y \rightarrow x$ then $g=\left(V_{0}^{f} \cup\{y\} ; V_{1}^{f}-\{y\} ; V_{2}^{f}\right)$ is a TRDF on D with $w(g)=w(f)-1$.

Observation 3. Let $D$ be a digraph and $f=\left(V_{0}, V_{1}, V_{2}\right)$ a $\gamma_{R}^{*}(D)$-function.

(i) If $v \in V(D)$ and $d^{+}(v) d^{-}(v)=0$ then $f(v) \neq 0$.

(ii) If $x, y, z \in V_{1}, x \rightarrow y$ and $y \rightarrow x$ then $y \not \rightarrow z$ or $z \not \rightarrow y$ holds.

(iii) If $x \in V_{1}$ then at least one of the sets $N^{+}(x) \cap V_{2}$ and $N^{-}(x) \cap V_{2}$ is empty.

(iv) $\left|V_{2}\right| \leq\left|V_{0}\right|$.

We will say that a digraph $D$ is a twin Roman digraph if $\gamma_{R}^{*}(D)=2 \gamma^{*}(D)$.

Observation 4. A digraph $D$ is a twin Roman digraph if and only if it has a $\gamma_{R}^{*}(D)$-function $f=\left(V_{0}, V_{1}, V_{2}\right)$ with $V_{1}=\varnothing$.

Proof. Let $D$ be a twin Roman digraph, and let $S$ be a $\gamma^{*}(D)$-set of $D$. Then $f=(V(D)-S, \varnothing, S)$ is a TRDF on $D$ such that $\omega(f)=2|S|=2 \gamma^{*}(D)=\gamma_{R}^{*}(G)$, and therefore $f$ is a $\gamma_{R}^{*}(D)$-function with $V_{1}=\varnothing$.

Conversely, let $f=\left(V_{0}, V_{1}, V_{2}\right)$ be a $\gamma_{R}^{*}(D)$-function with $V_{1}=\varnothing$ and thus $\gamma_{R}^{*}(D)=2\left|V_{2}\right|$. Then $V_{2}$ is also a twin dominating set of $D$ implying that $2 \gamma^{*}(D) \leq$ $2\left|V_{2}\right|=\gamma_{R}^{*}(D)$. Applying (1.1), we obtain the identity $\gamma_{R}^{*}(D)=2 \gamma^{*}(D)$, i.e. $D$ is a twin Roman digraph.

\section{BASIC PROPERTIES AND BOUNDS ON THE TWIN ROMAN DOMINATION NUMBER}

First we characterize the digraphs $D$ with the properties that $\gamma_{R}^{*}(D)=2, \gamma_{R}^{*}(D)=$ $3, \gamma_{R}^{*}(D)=4$ or $\gamma_{R}^{*}(D)=5$.

Proposition 1. (i) For a digraph $D$ of order $n \geq 2, \gamma_{R}^{*}(D)=2$ if and only if $n=2$ or there is a vertex $v$ with $d^{+}(v)=d^{-}(v)=n-1$.

(ii) For a digraph $D$ of order $n \geq 3, \gamma_{R}^{*}(D)=3$ if and only if $D$ has no vertex $v$ with $d^{+}(v)=d^{-}(v)=n-1$. In addition $(a) n=3$ or $(b) D$ has a vertex $v$ with $\left|N^{+}(v) \cap N^{-}(v)\right|=n-2$.

(iii) For a digraph $D$ of order $n \geq 4, \gamma_{R}^{*}(D)=4$ if and only if $\left|N^{+}(v) \cap N^{-}(v)\right| \leq$ $n-3$ for any vertex $v \in V(D)$. In addition, $(a) n=4$ or $(b)$ there is a vertex $v$ with $\left|N^{+}(v) \cap N^{-}(v)\right|=n-3$ or $(c)$ there are two vertices $u, v \in V(D)$ such that $\left(N_{D}^{+}(u) \cup N_{D}^{+}(v)\right) \cap\left(N_{D}^{-}(u) \cap N_{D}^{-}(v)\right)=V(D)-\{u, v\}$.

(iv) For a digraph $D$ of order $n \geq 5, \gamma_{R}^{*}(D)=5$ if and only if $\left|N^{+}(v) \cap N^{-}(v)\right| \leq$ $n-4$ for any vertex $v \in V(D)$ and $\left|\left(N_{D}^{+}(x) \cup N_{D}^{+}(y)\right) \cap\left(N_{D}^{-}(x) \cup N_{D}^{-}(y)\right)\right| \leq$ $n-3$ for all pairs of vertices $x, y \in V(D)$. In addition, $(a)$ there are two vertices $u, v \in V(D)$ such that $\left|\left(N_{D}^{+}(u) \cup N_{D}^{+}(v)\right) \cap\left(N_{D}^{-}(u) \cup N_{D}^{-}(v)\right)\right|=n-3$ 
or (b) $n=5$ or (c) D contains a vertex $w$ with $\left|N^{+}(w) \cap N^{-}(w)\right|=n-4$ and the induced subdigraph $H=D\left[V(D)-\left(N^{+}[w] \cap N^{-}[w]\right)\right]$ does not contain a vertex $x$ with $\left|N_{H}^{+}(x) \cap N_{H}^{-}(x)\right|=2$.

Proof. Since the proof of (i) is clear, we omit it.

(ii) Let $D$ have no vertex $v$ with $d^{+}(v)=d^{-}(v)=n-1$, then it follows from (i) that $\gamma_{R}^{*}(D) \geq 3$. The other two assumptions show that $\gamma_{R}^{*}(D) \leq 3$, and so we obtain $\gamma_{R}^{*}(D)=3$.

Conversely, assume that $\gamma_{R}^{*}(D)=3$. It follows from (i) that $D$ has no vertex $v$ with $d^{+}(v)=d^{-}(v)=n-1$. Let $f=\left(V_{0}, V_{1}, V_{2}\right)$ be a $\gamma_{R}^{*}(D)$-function. If $V_{2}=\varnothing$, then $\left|V_{1}\right|=3=n$ and thus (a) holds. If $V_{2} \neq \varnothing$, then $\left|V_{1}\right|=\left|V_{2}\right|=1$. Suppose $V_{2}=\{v\}$. Then $(u, v),(v, u) \in A(D)$ for each $u \in V_{0}$ and hence $\left|N^{+}(v) \cap N^{-}(v)\right|=n-2$. Thus condition (b) is proved.

(iii) Since $\left|N^{+}(v) \cap N^{-}(v)\right| \leq n-3$ for any vertex $v \in V(D)$, we deduce from (i) and (ii) that $\gamma_{R}^{*}(D) \geq 4$. The other three assumptions show that $\gamma_{R}^{*}(D) \leq 4$, and so we obtain $\gamma_{R}^{*}(D)=4$.

Conversely, assume that $\gamma_{R}^{*}(D)=4$. It follows from (i) and (ii) that $\mid N^{+}(v) \cap$ $N^{-}(v) \mid \leq n-3$ for any vertex $v \in V(D)$. Let $f=\left(V_{0}, V_{1}, V_{2}\right)$ be a $\gamma_{R}^{*}(D)$-function. If $V_{2}=\varnothing$, then $n=\left|V_{1}\right|=4$ and so (a) holds. We distinguish two cases.

Case 1. Assume that $\left|V_{2}\right|=1$ and $\left|V_{1}\right|=2$. If $V_{2}=\{v\}$, then we deduce that $\left|N^{+}(v) \cap N^{-}(v)\right|=n-3$ and the condition (b) holds.

Case 2. Assume that $\left|V_{2}\right|=2$. If $V_{2}=\{u, v\}$, then we conclude that $\left(N_{D}^{+}(u) \cup\right.$ $\left.N_{D}^{+}(v)\right) \cap\left(N_{D}^{+}(u) \cup N_{D}^{+}(v)\right)=V(D)-\{u, v\}$, and we obtain condition (c).

(iv) By (i), (ii), (iii), the conditions $\left|N^{+}(v) \cap N^{-}(v)\right| \leq n-4$ for any vertex $v \in$ $V(D)$ and $\left|\left(N_{D}^{+}(x) \cup N_{D}^{+}(y)\right) \cap\left(N_{D}^{-}(x) \cup N_{D}^{-}(y)\right)\right| \leq n-3$ for all pairs of vertices $x, y \in V(D)$ imply that $\gamma_{R}^{*}(D) \geq 5$. The other three assumptions show that $\gamma_{R}^{*}(D) \leq$ 5 , and so we obtain $\gamma_{R}^{*}(D)=5$.

Conversely, assume that $\gamma_{R}^{*}(D)=5$. Using (i), (ii) and (iii), we can see that $\left|N^{+}(v) \cap N^{-}(v)\right| \leq n-4$ for any vertex $v \in V(D)$ and $\mid\left(N_{D}^{+}(x) \cup N_{D}^{+}(y)\right) \cap\left(N_{D}^{-}(x)\right.$ $\left.\cup N_{D}^{-}(y)\right) \mid \leq n-3$ for all pairs of vertices $x, y \in V(D)$. Let $f=\left(V_{0}, V_{1}, V_{2}\right)$ a $\gamma_{R}^{*}(D)$-function. If $V_{2}=\varnothing$, then $\left|V_{1}\right|=5$ and thus $n=5$. Again, we distinguish two cases.

Case 1. Assume that $\left|V_{2}\right|=1$ and $\left|V_{1}\right|=3$. If $V_{2}=\{w\}$, then we deduce that $\left|N^{+}(w) \cap N^{-}(w)\right|=n-4$. Let $\{a, b, c\}=V(D)-\left(N^{+}[w] \cap N^{-}(w)\right)$. If $H=$ $D[\{a, b, c\}]$ contains a vertex $x$ with $\left|N_{H}^{+}(x) \cap N_{H}^{-}(x)\right|=2$, then we have condition (a). If $D[\{a, b, c\}]$ does not contain a vertex $x$ with $\left|N_{H}^{+}(x) \cap N_{H}^{-}(x)\right|=2$, then we have condition (c).

Case 2. Assume that $\left|V_{2}\right|=2$ and $\left|V_{1}\right|=1$. If $V_{2}=\{u, v\}$, then it follows that $\left|\left(N_{D}^{+}(u) \cup N_{D}^{+}(v)\right) \cap\left(N_{D}^{-}(u) \cup N_{D}^{-}(v)\right)\right|=n-3$ and condition (a) is proved.

Corollary 1. For any oriented graph $D$ of order $n \geq 4, \gamma_{R}^{*}(D) \geq 4$. 
Theorem 2. Let $D$ be a digraph of order $n$, maximum outdegree $\Delta^{+} \geq 1$ and maximum indegree $\Delta^{-}$. Then

$$
\gamma_{R}^{*}(D) \geq \max \left\{\left\lceil\frac{2 n}{\Delta^{+}+1}\right\rceil,\left\lceil\frac{2 n}{\Delta^{-}+1}\right\rceil\right\} .
$$

Proof. We only prove $\gamma_{R}^{*}(D) \geq\left\lceil(2 n) /\left(\Delta^{+}+1\right)\right\rceil$, as $\gamma_{R}^{*}(D) \geq\left\lceil(2 n) /\left(\Delta^{-}+1\right)\right\rceil$ can be proved similarly. Let $f=\left(V_{0}, V_{1}, V_{2}\right)$ be a $\gamma_{R}^{*}(D)$-function. Then $\gamma_{R}^{*}(D)=$ $\left|V_{1}\right|+2\left|V_{2}\right|$ and $n=\left|V_{0}\right|+\left|V_{1}\right|+\left|V_{2}\right|$. Since each vertex of $V_{0}$ has at least one in-neighbor in $V_{2}$, we observe that $\left|V_{0}\right| \leq \Delta^{+}\left|V_{2}\right|$. Since $\Delta^{+} \geq 1$, we deduce that

$$
\begin{aligned}
\left(\Delta^{+}+1\right) \gamma_{R}^{*}(D) & =\left(\Delta^{+}+1\right)\left(\left|V_{1}\right|+2\left|V_{2}\right|\right)=\left(\Delta^{+}+1\right)\left|V_{1}\right|+2\left|V_{2}\right|+2 \Delta^{+}\left|V_{2}\right| \\
& \geq\left(\Delta^{+}+1\right)\left|V_{1}\right|+2\left|V_{2}\right|+2\left|V_{0}\right|=2 n+\left(\Delta^{+}-1\right)\left|V_{1}\right| \geq 2 n .
\end{aligned}
$$

This inequality chain leads to $\gamma_{R}^{*}(D) \geq\left\lceil(2 n) /\left(\Delta^{+}+1\right)\right\rceil$.

If $K_{n}^{*}$ is the complete digraph of order $n \geq 2$, then Proposition 1 (i) implies that $\gamma_{R}^{*}\left(K_{n}^{*}\right)=2$. If $K_{n, n}^{*}$ is the complete bipartite digraph with $n \geq 4$, then it follows from Theorem 2 that $\gamma_{R}^{*}\left(K_{n, n}^{*}\right) \geq 4$. Now it is easy to see that $\gamma_{R}^{*}\left(K_{n, n}^{*}\right)=4$. These examples show that Theorem 2 is sharp.

If $D$ is the empty digraph of order $n$, then clearly $\gamma_{R}^{*}(D)=n$. Therefore Theorem 2 yields to the next result immediately.

Corollary 2. Let $D$ be a digraph of order $n$. If $\gamma_{R}^{*}(D)<n$, then $\Delta^{+}(D) \geq 2$ and $\Delta^{-}(D) \geq 2$.

Let $C_{n}^{*}$ be the digraph of order $n \geq 3$ with vertex set $\left\{v_{1}, v_{2}, \ldots, v_{n}\right\}$ such that $v_{i} \rightarrow$ $v_{i+1}, v_{i+1} \rightarrow v_{i}$ for $1 \leq i \leq n-1, v_{n} \rightarrow v_{1}$ and $v_{1} \rightarrow v_{n}$. Now it is straightforward to verify that $\gamma_{R}^{*}\left(C_{n}^{*}\right)=\lceil(2 n) / 3\rceil<n$ for $n \geq 3$. The digraph $C_{n}^{*}$ demonstrates that $\Delta^{+}(D)=2$ and $\Delta^{-}(D)=2$ in Corollary 2 is possible. In addition, this is a further example showing the sharpness of Theorem 2 .

Proposition 2. Let $D$ be a digraph of order $n$, maximum out-degree $\Delta^{+}$and maximum in-degree $\Delta^{-}$. If $\Delta^{+}+\Delta^{-} \geq n+3$, then $\gamma_{R}^{*}(D)<n$.

Proof. Let $d^{+}(v)=\Delta^{+}$.

First we assume that $d^{-}(v)=\Delta^{-}$. In this case the condition $\Delta^{+}+\Delta^{-} \geq n+3$ leads to $\left|N^{+}(v) \cap N^{-}(v)\right| \geq 4$. Then the function $f=\left(N^{+}(v) \cap N^{-}(v), V(D)-\right.$ $\left.\left(\left(N^{+}(v) \cap N^{-}(v)\right) \cup\{v\}\right),\{v\}\right)$ is a TRDF on $D$ of weight $\omega(f) \leq n-3$ and thus $\gamma_{R}^{*}(D) \leq n-3$.

Second we assume that $d^{-}(u)=\Delta^{-}$for a vertex $u \neq v$. The condition $\Delta^{+}+$ $\Delta^{-} \geq n+3$ implies that $\left|N^{+}(v) \cap N^{-}(u)\right| \geq 3$. Therefore the function $f=\left(N^{+}(v) \cap\right.$ $\left.N^{-}(u), V(D)-\left(\left(N^{+}(v) \cap N^{-}(u)\right) \cup\{u, v\}\right),\{u, v\}\right)$ is a TRDF on $D$ of weight $\omega(f) \leq n-1$ and thus $\gamma_{R}^{*}(D) \leq n-1$. This completes the proof. 
Let $H$ be the digraph with vertex set $\left\{v, u_{1}, u_{2}, \ldots, u_{n-1}\right\}$ with $n \geq 5$ such that $v \rightarrow$ $u_{i}$ for $i=1,2, \ldots, n-1, u_{2} \rightarrow u_{1}$ and $u_{3} \rightarrow u_{1}$. Then $\Delta^{+}(H)+\Delta^{-}(H)=n-2$ and $\gamma_{R}^{*}(H)=n$. This example demonstrates that the condition $\Delta^{+}+\Delta^{-} \geq n+3$ in Proposition 2 is best possible in some sense.

Proposition 3. Let $D$ be a digraph. The following statements are equivalent.

(i) $\gamma^{*}(D)=\gamma_{R}^{*}(D)$.

(ii) $\gamma^{*}(D)=|V(D)|$.

(iii) There is no a directed path of length 2 in $D$.

Proof. (i) $\Rightarrow$ (ii): Let $\gamma^{*}(D)=\gamma_{R}^{*}(D)$. Then for any $\gamma_{R}^{*}(D)$-function $f=$ $\left(V_{0}, V_{1}, V_{2}\right)$ on $D$ we have $\gamma^{*}(D) \leq\left|V_{1}\right|+\left|V_{2}\right| \leq\left|V_{1}\right|+2\left|V_{2}\right|=\gamma_{R}^{*}(D)$. Hence $V_{2}=\varnothing$ implying that $V_{0}=\varnothing$. Therefore $\gamma^{*}(D)=\gamma_{R}^{*}(D)=\left|V_{1}\right|=|V(D)|$.

(ii) $\Rightarrow$ (i): The result follows immediately by (1.1) and (1.2).

(ii) $\Leftrightarrow$ (iii): Obvious.

Proposition 4. If $D$ is a digraph on $n$ vertices, then $\gamma_{R}^{*}(D) \geq \min \left\{n, \gamma^{*}(D)+1\right\}$.

Proof. If $\gamma_{R}^{*}(D)=n$, then the result is immediate. Assume now that $\gamma_{R}^{*}(D)<n$, and suppose to the contrary that $\gamma_{R}^{*}(D) \leq \gamma^{*}(D)$. By (1.1) we have $\gamma_{R}^{*}(D)=\gamma^{*}(D)$. Now Proposition 3 implies $\gamma_{R}^{*}(D)=n$, a contradiction.

Proposition 5. Let $D$ be a digraph of order $n \neq 3$ with $\delta(D) \geq 1$. Then $\gamma_{R}^{*}(D)=$ $\gamma^{*}(D)+1$ if and only if there is a vertex $v \in V(D)$ with $\left|N^{+}(v) \cap N^{-}(v)\right|=n-$ $\gamma^{*}(D)$.

Proof. Let $D$ have a vertex $v$ with $\left|N^{+}(v) \cap N^{-}(v)\right|=n-\gamma^{*}(D)$. Then clearly $f=\left(N^{+}(v) \cap N^{-}(v), V(D)-\left(N^{+}[v] \cap N^{-}[v]\right),\{v\}\right)$ is a TRDF on $D$ of weight $\gamma^{*}(D)+1$. Hence $\gamma_{R}^{*}(D) \leq \gamma^{*}(D)+1$, and the result follows by Proposition 4 .

Conversely, let $\gamma_{R}^{*}(D)=\gamma^{*}(D)+1$ and let $f=\left(V_{0}, V_{1}, V_{2}\right)$ be a $\gamma_{R}^{*}(D)$-function. Then either (1) $\left|V_{1}\right|=\gamma^{*}(D)+1$ and $\left|V_{2}\right|=0$ or (2) $\left|V_{1}\right|=\gamma^{*}(D)-1$ and $\left|V_{2}\right|=1$.

In case (1), since $\left|V_{2}\right|=0$, we have $\left|V_{0}\right|=0$. Hence $n=\gamma^{*}(D)+1$. It follows from Theorem 1 that $n=\gamma^{*}(D)+1 \leq \frac{2 n}{3}+1$, a contradiction when $n \geq 4$. If $n=2$, then the hypothesis $\delta(D) \geq 1$ implies that $D$ consists of two vertices $x$ and $y$ such that $x \rightarrow y \rightarrow x$ and thus $\left|N^{+}(x) \cap N^{-}(x)\right|=1=2-1=n-\gamma^{*}(D)$.

In case (2), let $V_{2}=\{v\}$. Then $(v, u),(u, v) \in A(D)$ for each $u \in V_{0}$. Since $N^{+}(v) \cap N^{-}(v) \cap V_{1}=\varnothing$, we obtain $\left|N^{+}(v) \cap N^{-}(v)\right|=\left|V_{0}\right|=n-\left|V_{1}\right|-\left|V_{2}\right|=$ $n-\gamma^{*}(D)$.

Proposition 6. Let $D$ be a digraph on $n \geq 7$ vertices with $\delta(D) \geq 1$. Then $\gamma_{R}^{*}(D)=\gamma^{*}(D)+2$ if and only if:

(i) D does not have a vertex $v$ with with $\left|N^{+}(v) \cap N^{-}(v)\right|=n-\gamma^{*}(D)$.

(ii) either $D$ has a vertex $v$ with with $\left|N^{+}(v) \cap N^{-}(v)\right|=n-\gamma^{*}(D)-1$ or $D$ contains two vertices $v, w$ such that

$$
\left|\left(N^{+}[v] \cup N^{+}[w]\right) \cap\left(N^{-}[v] \cup N^{-}[w]\right)\right|=n-\gamma^{*}(D)+2 .
$$


Proof. Let $\gamma_{R}^{*}(D)=\gamma^{*}(D)+2$. It follows from Proposition 5 that $D$ does not have a vertex $v$ with $\left|N^{+}(v) \cap N^{-}(v)\right|=n-\gamma^{*}(D)$. Let $f=\left(V_{0}, V_{1}, V_{2}\right)$ be a $\gamma_{R}^{*}(D)$-function. Then either (1) $\left|V_{1}\right|=\gamma^{*}(D)+2$ and $\left|V_{2}\right|=0$, (2) $\left|V_{1}\right|=\gamma^{*}(D)$ and $\left|V_{2}\right|=1$, or (3) $\left|V_{1}\right|=\gamma^{*}(D)-2$ and $\left|V_{2}\right|=2$.

In case (1), we have $\left|V_{0}\right|=0$. Then $V(D)=V_{1}$. By Theorem 1 , we have $n=$ $\gamma^{*}(D)+2 \leq \frac{2 n}{3}+2$ which leads to a contradiction because $n \geq 7$.

In case (2), let $V_{2}=\{v\}$. Obviously $(v, u) \in A(D)$ and $(u, v) \in A(D)$ for each $u \in V_{0}$. Since for each $x \in V_{1},(v, u) \notin A(D)$ or $(u, v) \notin A(D)$, we obtain $\mid N^{+}(v) \cap$ $N^{-}(v) \mid=n-\gamma^{*}(D)-1$.

In case (3), let $V_{2}=\{v, w\}$. Since $(v, u) \in A(D)$ or $(w, u) \in A(D)$ and $(u, v) \in$ $A(D)$ or $(u, w) \in A(D)$ for each $u \in V_{0}$ and since $x \notin\left(N^{+}[v] \cup N^{+}[w]\right) \cap\left(N^{-}[v] \cup\right.$ $\left.N^{-}[w]\right)$ for each $x \in V_{0}$, we deduce that $\left|\left(N^{+}[v] \cup N^{+}[w]\right) \cap\left(N^{-}[v] \cup N^{-}[w]\right)\right|=$ $n-\left|V_{1}\right|=n-\left(\gamma^{*}(D)-2\right)=n-\gamma^{*}(D)+2$.

Conversely, let $D$ satisfy (i) and (ii). It follows from Proposition 5 and (i) that $\gamma_{R}^{*}(D) \geq \gamma^{*}(D)+2$. If $D$ has a vertex $v$ with $\left|N^{+}(v) \cap N^{-}(v)\right|=n-\gamma^{*}(D)-1$, then obviously $f=\left(N^{+}(v) \cap N^{-}(v), V(D)-\left(N^{+}[v] \cap N^{-}[v]\right),\{v\}\right)$ is a TRDF on $D$ of weight $\gamma^{*}(D)+2$ implying that $\gamma_{R}^{*}(D) \leq \gamma^{*}(D)+2$. If $D$ has two vertices $v, w$ such that $\left|\left(N^{+}[v] \cup N^{+}[w]\right) \cap\left(N^{-}[v] \cup N^{-}[w]\right)\right|=n-\gamma^{*}(D)+2$, then $f=\left(\left(N^{+}[v] \cup N^{+}[w]\right) \cap\left(N^{-}[v] \cup N^{-}[w]\right), V(D)-\left(N^{+}[v] \cup N^{+}[w]\right) \cap\left(N^{-}[v] \cup\right.\right.$ $\left.\left.N^{-}[w]\right),\{v, w\}\right)$ is a TRDF on $D$ of weight $\gamma^{*}(D)+2$ and the result follows again. This completes the proof.

\section{TWIN ROMAN DOMINATION IN ORIENTED GRAPHS}

An orientation of a graph $G$ is a digraph $D$ obtained from $G$ by choosing an orientation $(x \rightarrow y$ or $y \rightarrow x)$ for every edge $x y \in E(G)$. Clearly, two distinct orientations of a graph can have distinct twin domination numbers. Motivated by this observation Chartrand et al. [3] introduced the concept of the lower orientable twin domination number $\operatorname{dom}^{*}(G)$ and the upper orientable twin domination number $\operatorname{DOM}^{*}(G)$ of a graph $G$, as

$$
\operatorname{dom}^{*}(G)=\min \left\{\gamma^{*}(D) \mid \mathrm{D} \text { is an orientation of } G\right\},
$$

and

$$
\operatorname{DOM}^{*}(G)=\max \left\{\gamma^{*}(D) \mid \mathrm{D} \text { is an orientation of } G\right\} .
$$

This concepts have been studied in [2].

Here, we propose similar concepts the lower orientable twin Roman domination number $\operatorname{dom}_{R}^{*}(G)$ and the upper orientable twin Roman domination number $\operatorname{DOM}_{R}^{*}(G)$ as follows.

$$
\operatorname{dom}_{R}^{*}(G)=\min \left\{\gamma_{R}^{*}(D) \mid \mathrm{D} \text { is an orientation of } G\right\},
$$


and

$$
\operatorname{DOM}_{R}^{*}(G)=\max \left\{\gamma_{R}^{*}(D) \mid \mathrm{D} \text { is an orientation of } G\right\} .
$$

Clearly $\operatorname{dom}_{R}^{*}(G) \leq \operatorname{DOM}_{R}^{*}(G) \leq n$ for every graph $G$ of order $n$.

Proposition 7. Let $G$ be a graph of order $n$ with at most one cycle. Then $\operatorname{dom}_{R}^{*}(G)=n$.

Proof. By (1.2), it is enough to prove $\gamma_{R}^{*}(\vec{G}) \geq n$. First let $G$ be not a cycle. We proceed by induction on $n$. The result can be easily verified for all graphs with at most 3 vertices. Hence, suppose that $n \geq 4$ and the result is true for all graphs of order less than $n$. Let $G$ be a graph of order $n$. By assumption $G$ has an end vertex, say $x$. Let $\vec{G}$ be an orientation of $G$. Then obviously for any $\gamma_{R}^{*}(\vec{G})$-function $f=\left(V_{0}, V_{1}, V_{2}\right)$, $f(x) \neq 0$. If $f(x)=1$ then $g=\left(V_{0}, V_{1}-\{x\}, V_{2}\right)$ is a TRDF on $\vec{G}-\{x\}$ and it follows from the induction hypothesis that $\gamma_{R}^{*}(\vec{G})=\omega(g)+1 \geq \gamma_{R}^{*}(\vec{G}-\{x\})+1 \geq$ $n$, as desired. Now let $f(x)=2$. Then $f(y)=0$, where $y$ is the support vertex of $x$ in $G$. This implies that the function $h=\left(V_{0}-\{y\} ; V_{1} \cup\{y\} ; V_{2}-\{x\}\right)$ is a TRDF on $\vec{G}-\{x\}$ with $\omega(h)=\gamma_{R}^{*}(D)-1$. Now the result follows by the induction hypothesis as above.

Now let $G=C_{n}$ and let $\vec{G}$ be an orientation of $G$. Assume to the contrary that $\gamma_{R}^{*}(D)<n$. Suppose $f=\left(V_{0}^{f}, V_{1}^{f}, V_{2}^{f}\right)$ is a $\gamma_{R}^{*}(\vec{G})$-function. Then both of $V_{0}^{f}$ and $V_{2}^{f}$ are nonempty. Hence (a) each vertex in $V_{0}^{f}$ has exactly 2 neighbors and they both are in $V_{2}^{f}$, and (b) each vertex in $V_{2}^{f}$ has at most 1 neighbor not in $V_{0}^{f}$. From (a) and (b) it immediately follows that $\left|V_{0}^{f}\right| \leq\left|V_{2}^{f}\right|$. Hence $\gamma_{R}^{*}(\vec{G})=\left|V_{1}^{f}\right|+2\left|V_{2}^{f}\right|=$ $\left|V_{0}^{f}\right|+\left|V_{1}^{f}\right|+\left|V_{2}^{f}\right|=n$ and the proof is completed.

The next results are immediate consequences of Proposition 7.

Corollary 3. For $n \geq 1, \operatorname{dom}_{R}^{*}\left(K_{1, n}\right)=n$.

Corollary 4. $\operatorname{dom}_{R}^{*}\left(C_{n}\right)=\operatorname{dom}_{R}^{*}\left(P_{n}\right)=\operatorname{Dom}_{R}^{*}\left(C_{n}\right)=\operatorname{Dom}_{R}^{*}\left(P_{n}\right)=n$.

Proposition 8. For any graph $G$ of order $n \geq 4$ with clique number $c \geq 4$, $\operatorname{dom}_{R}^{*}(G) \leq n-c+4$.

Proof. Let $S=\left\{v_{1}, v_{2}, \ldots, v_{c}\right\}$ be a clique in $G$. Let $\vec{G}$ be an orientation of $G$ such that the edges are oriented from $v_{1}$ to $v_{2}, v_{3}, \ldots, v_{c}$ and from $v_{3}, v_{4}, \ldots, v_{c}$ to $v_{2}$ and the other edges oriented arbitrary. Then $f=\left(\left\{v_{3}, v_{4}, \ldots, v_{c}\right\}, V(G)-S,\left\{v_{1}, v_{2}\right\}\right)$ is a twin Roman dominating function of $\vec{G}$ which yields $\operatorname{dom}_{R}^{*}(G) \leq n-c+4$.

An independent set is a set of vertices that no two of which are adjacent. A maximum independent set is an independent set of largest possible size. This size is called the independence number of $\mathrm{G}$, and denoted by $\alpha(G)$. 
Proposition 9. For any graph $G$ of order $n \geq 4$ with $\delta(G) \geq 2$, $\operatorname{dom}_{R}^{*}(G) \leq 2(n-$ $\alpha(G))$.

Proof. Let $S=\left\{v_{1}, v_{2}, \ldots, v_{\alpha(G)}\right\}$ be an independent set of $G$. Since $S$ is independent and $\delta(G) \geq 2$, each $v_{i}$ has two neighbors $u_{i}, w_{i}$ in $V-S$. Let $\vec{G}$ be an orientation of $G$ such that $\left(v_{i}, u_{i}\right),\left(w_{i}, v_{i}\right) \in A(\vec{G})$. Then the function $f=(S, \varnothing, V-S)$ is a twin Roman dominating function of $\vec{G}$ that implies that $\operatorname{dom}_{R}^{*}(G) \leq 2(n-$ $\alpha(G))$.

The next results are immediate consequences of Corollary 1 and Propositions 8 and 9 .

Corollary 5. For $n \geq 4, \operatorname{dom}_{R}^{*}\left(K_{n}\right)=4$.

Corollary 6. For $n \geq 2, \operatorname{dom}_{R}^{*}\left(K_{2, n}\right)=4$.

Theorem 3. ([2]) For $r \geq s \geq 3$,

$$
\operatorname{dom}^{*}\left(K_{r, s}\right)=\left\{\begin{array}{lll}
3 & \text { if } & s=3 \\
4 & \text { if } & s \geq 4 .
\end{array}\right.
$$

Proposition 10. For every two integers $r \geq s \geq 3$,

$$
\operatorname{dom}_{R}^{*}\left(K_{r, s}\right)=\left\{\begin{array}{lll}
5 & \text { if } & s=3 \\
6 & \text { if } & s=4 \\
7 & \text { if } & s=5 \\
8 & \text { if } & s \geq 6
\end{array}\right.
$$

Proof. Let $G=K_{r, s}$ and let $X=\left\{x_{1}, x_{2}, \ldots, x_{s}\right\}$ and $Y=\left\{y_{1}, y_{2}, \ldots, y_{r}\right\}$ be the partite sets of $G$. Consider the following cases.

Case 1. $s=3$.

It follows from Propositions 4, 5 and Theorem 3 that $\gamma_{R}^{*}(G) \geq \gamma^{*}(G)+2=5$. Let $\vec{G}$ be an orientation of $G$ such that $\left(x_{1}, y_{i}\right),\left(y_{i}, x_{2}\right) \in A(\vec{G})$ for each $i$. Clearly, $g=\left(Y,\left\{x_{3}\right\},\left\{x_{1}, x_{2}\right\}\right)$ is a TRDF of $\vec{G}$ that implies $\gamma_{R}^{*}(G) \leq 5$. Hence $\gamma_{R}^{*}(G)=5$.

Case 2. $s=4$.

Using an argument similar to that described in Case 1, we obtain $\gamma_{R}^{*}(G)=6$.

Case 3. $s=5$.

Suppose $\vec{G}$ is an orientation of $G$ such that $\left(x_{1}, y_{i}\right),\left(y_{i}, x_{2}\right) \in A(\vec{G})$ for each $i$. Obviously, $g=\left(Y,\left\{x_{3}, x_{4}, x_{5}\right\},\left\{x_{1}, x_{2}\right\}\right)$ is a TRDF of $\vec{G}$ implying that $\gamma_{R}^{*}(G) \leq$ 7. Let $D$ be an arbitrary orientation of $G$. Since $G$ has no cycle of length 2 and for any two vertices $u, v \in V(G),\left|\left(N^{+}[v] \cup N^{+}[u]\right) \cap\left(N^{-}[v] \mid \cup N^{-}[u]\right)\right| \leq n-$ $3=|V(G)|-\gamma^{*}(G)+1$, we deduce from Propositions 4, 5, 6 and Theorem 3 that $\gamma_{R}^{*}(G) \geq \gamma^{*}(G)+3=7$. Thus $\gamma_{R}^{*}(G)=7$.

Case 4. $s \geq 6$.

It follows from Theorem 3 and (1.1) that $\gamma_{R}^{*}(G) \leq 8$. Let $D$ be an arbitrary orientation 
of $G$ and $f=\left(V_{0}, V_{1}, V_{2}\right)$ a $\gamma_{R}^{*}(D)$-function. Since $\gamma_{R}^{*}(G) \leq 8$, we deduce that $V_{0} \neq \varnothing$. If $V_{0} \cap X \neq \varnothing$ and $V_{0} \cap Y \neq \varnothing$, then we must have $\left|V_{2} \cap Y\right| \geq 2$ and $\left|V_{2} \cap X\right| \geq 2$ that implies $\gamma_{R}^{*}(G) \geq 8$ as desired. Now let, without loss of generality, $V_{0} \cap X=\varnothing$. Then $V_{0} \cap Y \neq \varnothing$ that implies $\left|V_{2} \cap X\right| \geq 2$ and hence $\gamma_{R}^{*}(D) \geq$ $4+|X|-2=s+2 \geq 8$. Thus $\gamma_{R}^{*}(G)=8$ and the proof is completed.

Proposition 11. Let $G=K_{m_{1}, m_{2}, \ldots, m_{r}}(r \geq 3)$ be the complete $r$-partite graph with $1 \leq m_{1} \leq m_{2} \leq \ldots \leq m_{r}$. Then

$$
\operatorname{dom}_{R}^{*}\left(K_{\left.m_{1}, m_{2}, \ldots, m_{r}\right)}\right)=\left\{\begin{array}{lll}
4 & \text { if } & m_{1}=\cdots=m_{r}=1, \\
4 & \text { if } & m_{1}=m_{2}=1 \text { or } m_{i}=2 \text { for some } i, \\
5 & \text { if } \quad m_{1}=3 \text { or } m_{1}=1 \text { and } m_{2}=3, \\
6 & \text { if } \quad m_{1} \geq 4
\end{array}\right.
$$

Proof. Let $G=K_{m_{1}, m_{2}, \ldots, m_{r}}$ and let $X_{1}=\left\{x_{1}, x_{2}, \ldots, x_{m_{1}}\right\}, X_{2}=\left\{y_{1}, y_{2}, \ldots\right.$, $\left.y_{m_{2}}\right\}, X_{3}=\left\{z_{1}, z_{2}, \ldots, z_{m_{3}}\right\}, X_{4}, X_{5}, \ldots, X_{r}$ be the partite sets of $G$.

If $m_{1}=\cdots=m_{r}=1$ then $G=K_{n}$ and by Corollary 5, we have $\gamma_{R}^{*}(G)=4$. If $m_{1}=m_{2}=1$, then let $\vec{G}$ be an orientation of $G$ such that $\left(x_{1}, x\right),\left(x, y_{1}\right) \in A(\vec{G})$ for each $x \in V(G)-\left\{x_{1}, y_{1}\right\}$. Obviously, $g=\left(V(D)-\left\{x_{1}, y_{1}\right\}, \varnothing,\left\{x_{1}, y_{1}\right\}\right)$ is a TRDF of $\vec{G}$ implying that $\gamma_{R}^{*}(G)=4$ by Corollary 1 . If $m_{i}=2$ for some $i$, say $i=2$, then assume $\vec{G}$ is an orientation of $G$ such that $\left(y_{1}, x\right),\left(x, y_{2}\right) \in A(\vec{G})$ for each $x \in V(G)-\left\{y_{1}, y_{2}\right\}$. Clearly, $g=\left(V(D)-\left\{y_{1}, y_{2}\right\}, \varnothing,\left\{y_{1}, y_{2}\right\}\right)$ is a TRDF of $\vec{G}$ that implies $\gamma_{R}^{*}(G)=4$ again. If $m_{1}=3$ or $m_{1}=1$ and $m_{2}=3$ then as Case 1 . in Proposition 10, we deduce that $\gamma_{R}^{*}(G)=5$.

Finally, let $m_{1} \geq 4$. It follows from Proposition 1 that $\gamma_{R}^{*}(G) \geq 6$. Let $\vec{G}$ be an orientation of $G$ such that $\left(x_{1}, x\right),\left(x, y_{1}\right) \in A(\vec{G})$ for each $x \in V(G)-\left\{x_{1}, y_{1}\right\}$, $\left(z_{1}, x_{i}\right) \in A(\vec{G})$ for $2 \leq i \leq m_{1}$ and $\left(y_{i}, z_{1}\right) \in A(\vec{G})$ for $2 \leq i \leq m_{2}$. It is easy to see that $g=\left(V(D)-\left\{x_{1}, y_{1}, z_{1}\right\}, \varnothing,\left\{x_{1}, y_{1}, z_{1}\right\}\right)$ is a TRDF of $\vec{G}$ which implies $\gamma_{R}^{*}(G) \leq 6$. Thus $\gamma_{R}^{*}(G)=6$ and the proof is completed.

Theorem 4. For $n \geq 9, \operatorname{dom}_{R}^{*}\left(W_{n+1}\right)=\left\lceil\frac{2 n}{3}\right\rceil+2$.

Proof. Let $W_{n+1}=x+C_{n}$ and $C_{n}=\left(v_{1}, v_{2}, \ldots, v_{n}\right)$. Let $\overrightarrow{W_{n+1}}$ be an orientation of $W_{n+1}$ such that $\left(v_{i}, x\right) \in A\left(\overrightarrow{W_{n+1}}\right)$ for each $i$ and $\left(v_{i}, v_{i-1}\right),\left(v_{i}, v_{i+1}\right) \in A\left(\overrightarrow{W_{n+1}}\right)$ for each $i \equiv 1(\bmod 3)$. It is easy to see that the function $f$ that assigns 2 to $x$ and $v_{i}$ for $i \equiv 1(\bmod 3), \varnothing$ to $v_{i-1}$ and $v_{i+1}$ for $i \equiv 1(\bmod 3)$ and 1 to the other vertices, is an TRDF of $\overrightarrow{W_{n+1}}$ that yields $\operatorname{dom}_{R}^{*}\left(W_{n+1}\right) \leq \gamma_{R}^{*}\left(\overrightarrow{W_{n+1}}\right) \leq\left\lceil\frac{2 n}{3}\right\rceil+2$.

Now let $D$ be any orientation of $W_{n+1}$ and let $f$ be an $\gamma_{R}^{*}(D)$-function. If $f(v) \leq 1$, then $f$ is a TRDF of $C_{n}$ and hence $\gamma_{R}^{*}(D)=\omega(f) \geq n \geq\left\lceil\frac{2 n}{3}\right\rceil+2$ by Corollary 4. Assume $f(v)=2$. Then the function $f$, restricted to $C_{n}$ is an RDF of 
$C_{n}$ and we deduce from Proposition 7 in [4] that $\gamma_{R}^{*}(D)=\omega(f) \geq\left\lceil\frac{2 n}{3}\right\rceil+2$. Thus $\operatorname{dom}_{R}^{*}\left(W_{n+1}\right) \geq\left\lceil\frac{2 n}{3}\right\rceil+2$ and the proof is completed.

Theorem 5 ([2]). For $n \geq 3$, $\operatorname{DOM}^{*}\left(W_{n+1}\right) \geq n-1$.

Theorem 6. For $n \geq 4, \mathrm{DOM}_{R}^{*}\left(W_{n+1}\right)=n+1$.

Proof. Let $D$ be an orientation of $W_{n+1}$ for which $\operatorname{DOM}^{*}\left(W_{n+1}\right)=\gamma^{*}\left(W_{n+1}\right) \geq$ $n-1$. Assume that $f=\left(V_{0}, V_{1}, V_{2}\right)$ is a $\gamma_{R}^{*}(D)$-function. If $V_{0}=\varnothing$, then $V_{2}=\varnothing$ and we have $\gamma_{R}^{*}(D)=\left|V_{1}\right|=n+1$. Let $V_{0} \neq \varnothing$. To in-dominate and out-dominate of each vertex $u \in V_{0}$, we must have $\left|V_{2}\right| \geq 2$. Then $\gamma_{R}^{*}(D)=\left|V_{1}\right|+2\left|V_{2}\right| \geq 2+$ $\left|V_{1}\right|+\left|V_{2}\right| \geq 2+\gamma^{*}(D) \geq n+1$. It follows that $\operatorname{DOM}_{R}^{*}\left(W_{n+1}\right)=n+1$.

\section{REFERENCES}

[1] T. Araki, "The k-tuple twin domination in de Bruijn and Kautz digraphs," Discrete Math., vol. 308, pp. 6406-6413, 2008, doi: 10.1016/j.disc.2007.12.020.

[2] S. Arumugam, K. Ebadi, and L. Sathikala, "Twin domination and twin irredudance in digraphs," Appl. Anal. Discrete Math., vol. 7, pp. 275-284, 2013, doi: 10.2298/AADM130429007A.

[3] G. Chartrand, P. Dankelmann, M. Schultz, and H. C. Swart, "Twin domination in digraphs," Ars Combin., vol. 67, pp. 105-114, 2003.

[4] E. J. Cockayne, P. M. Dreyer Jr., S. M. Hedetniemi, and S. T. Hedetniemi, "Roman domination in graphs," Discrete Math., vol. 278, pp. 11-22, 2004, doi: 10.1016/j.disc.2003.06.004.

[5] M. Kamaraj and V. Hemalatha, "Directed Roman domination in digraphs," International Journal of Combinatorial Graph Theory and Applications, vol. 4, pp. 103-116, 2011.

[6] K. Muthu Pandian and M. Kamaraj, "Fractional twin domination number in digraphs," International Journal of Mathematical Archive, vol. 3, pp. 2375-2379, 2012.

[7] S. M. Sheikholeslami and L. Volkmann, "The Roman domination number of a digraph," Acta Univ. Apulensis Math. Inform., vol. 27, pp. 77-86, 2011.

[8] D. B. West, "Introduction to graph theory," Prentice-Hall, Inc, 2000.

Authors' addresses

H. Abdollahzadeh Ahangar

Department of Basic Science, Babol University of Technology, Babol, I.R. Iran

E-mail address: ha. ahangarenit.ac.ir

\section{J. Amjadi}

Azarbaijan Shahid Madani University, Department of Mathematics, Tabriz, I.R. Iran

E-mail address: j-amjadi@azaruniv.edu

\section{S.M. Sheikholeslami}

Azarbaijan Shahid Madani University, Department of Mathematics, Tabriz, I.R. Iran

E-mail address: s.m.sheikholeslami@azaruniv.edu

\section{Samodivkin}

Department of Mathematics, University of Architecture Civil Engineering and Geodesy, Hristo Smirnenski 1 Blv., 1046 Sofia, Bulgaria

E-mail address: vlsam_fte@uacg.bg 
L. Volkmann

RWTH Aachen University, Lehrstuhl II für Mathematik, 52056, Aachen, Germany

E-mail address: volkmemath2.rwth-aachen. de 